\title{
Supporting Information for 'Nonadiabatic
}

\section{Dynamics between Valence, Nonvalence and}

\section{Continuum Electronic States in a}

\section{Heteropolycyclic Aromatic Hydrocarbon'}

James N. Bull, ${ }^{* \dagger}$ Cate S. Anstöter ${ }^{\ddagger}$ Mark H. Stockett, ${ }^{\mp}$ Connor J. Clarke, ${ }^{\S}$ Jemma A. Gibbard, ${ }^{\S}$ Evan J. Bieske," and Jan R. R. Verlet ${ }^{\S}$

$\dagger$ School of Chemistry, Norwich Research Park, University of East Anglia, Norwich NR4 7 TJ, United Kingdom

$\ddagger$ Department of Chemistry, Temple University, Philadelphia, Pennsylvania 19122, United States

【Department of Physics, Stockholm University, Stockholm SE-106 91, Sweden $\S$ Department of Chemistry, Durham University, Durham DH1 3LE, United Kingdom ||School of Chemistry, University of Melbourne, Melbourne, VIC 3010, Australia E-mail: james.bull@uea.ac.uk 


\section{Experimental}

All experiments used a commercial sample of pyren-1-ol obtained from Sigma-Aldrich with purity $>99 \%$.

\section{Photodetachment action spectroscopy}

The photodetachment spectrum of $\mathrm{Py}^{-}$at $T \approx 300 \mathrm{~K}$ was recorded using a tandem ion mobility mass spectrometer coupled with laser excitation. ${ }^{1,2}$ Briefly, a methanoic solution with analyte concentration at $\approx 10^{-6} \mathrm{~mol} \mathrm{~L}^{-1}$ was loaded into a syringe, which was connected via a section of silica capillary (flow rate $\approx 10 \mu \mathrm{L} \mathrm{min}^{-1}$ ) to an electrospray source operating at $-3.3 \mathrm{kV}$. Electrosprayed ions were transferred through a heated capillary into a RF ion funnel, which gathered the ions and propelled them towards an electrostatic ion gate that injected $\approx 100 \mu$ s packets of ions at $40 \mathrm{~Hz}$ into the drift region. In the drift region, the ions were propelled by an electric field $\left(44 \mathrm{~V} \mathrm{~cm}^{-1}\right)$ through $\mathrm{N}_{2}+\approx 1 \% \mathrm{SF}_{6}$ buffer gas at a pressure of $\approx 6$ Torr. Midway along the drift region, a tunable light pulse from an EKSPLA NT342B optical parametric oscillator $\left(20 \mathrm{~Hz}, 2-3 \mathrm{~mJ} \mathrm{~cm}^{-2}\right.$ pulse $\left.^{-1}\right)$ was timed to intercept the Py1ion packet. Photodetached electrons were captured by $\mathrm{SF}_{6}$, generating $\mathrm{SF}_{6}^{-}(m / z 146){ }^{2}$ At the end of the drift region, an ion funnel collected the ions and introduced them into a differentially pumped octupole ion guide followed by a quadrupole mass filter before they reached a Channeltron ion detector. The detector was connected to a multichannel scaler that produced a histogram of ion counts against arrival time, providing an arrival time distribution. The photodetachment spectrum was constructed by integrating the $\mathrm{SF}_{6}^{-}$signal in the arrival time distribution for a given photon energy and normalizing with respect to light fluence and light-off $\mathrm{Py}^{-}{ }^{-}$signal.

It is important to note that $\mathrm{Py}^{-}$is photoactivated in a high pressure environment ( $\approx 6$ Torr $\mathrm{N}_{2}$ gas), such that slower processes are suppressed due to collisional quenching. The collision rate is $\approx 10^{9} \mathrm{~s}^{-1}$ at 6 Torr, although hundreds of collisions are required to 
quench the energy imparted through absorption of a visible photon. Under these conditions, rapid excited state processes occurring on sub-nanosecond timescales will tend to take place, e.g. autodetachment. On the other hand, slower ground state processes such as statistical dissociation or thermionic emission (statistical electron ejection over the microsecond to millisecond timescale ${ }^{3}$ ) are suppressed because of collisional deactivation. ${ }^{4,5}$ For Py1 ${ }^{-}$, no photodissociation was observed when the laser was tuned to the origin transition of the $\mathrm{S}_{1}$ band $(h \nu=2.60 \mathrm{eV})$ and with varying light fluence $\left(2-20 \mathrm{~mJ} \mathrm{~cm}^{-2}\right.$ pulse $\left.^{-1}\right)$. 


\section{Photodepletion action spectroscopy}

Photodepletion spectroscopy with provision for photodissociation on electrosprayed $\mathrm{Py}^{-}$ at $T \approx 300 \mathrm{~K}$ was performed using the Sep1 accelerator mass spectrometer at Aarhus University. ${ }^{6,7}$ The target molecule was dissolved in methanol with a trace amount of $\mathrm{NH}_{4} \mathrm{OH}$. Electrosprayed anions were accumulated in an octupole ion trap that was emptied every $25 \mathrm{~ms}$ ( $40 \mathrm{~Hz}$ repetition rate). The ion bunches were accelerated to a kinetic energy of $50 \mathrm{keV}$ and mass selected using a bending magnet. An OPO laser system (EKSPLA NT342A, $20 \mathrm{~Hz}$, 2-3 $\mathrm{mJ} \mathrm{cm}^{-2}$ pulse $^{-1}$, unfocused) interacted with every second ion bunch midway along a $2.5 \mathrm{~m}$ linear flight region. For a given wavelength of light, the difference in the number of counts between the 'laser-on' and 'laser-off' injections provided the photoinduced signal. Any photofragment ions could be separated using an electrostatic energy analyzer situated after the irradiation region and were detected with a channeltron. The photodepletion action spectrum recorded using the Sep1 instrument is shown in Figure S1. The spectrum is in close agreement with the photodetachment action spectrum.

In contrast to the photodetachment action spectrometer described earlier, anion irradiation in Sep1 occurs in a high vacuum region $\left(10^{-6}\right.$ Torr background pressure), allowing observation of processes occurring upto several hundred microseconds after photoactivation. For $\mathrm{Py}^{-}$, there was no evidence for photodissociation. The lack of photodissociation is consistent with the photoelectron spectroscopy experiments, which found no evidence for thermionic emission. Fluorescence will not be competitive on the ultrafast timescales characterised in the time-resolved experiments. We conclude that the photoexcited anions decompose exclusively through autodetachment processes. 


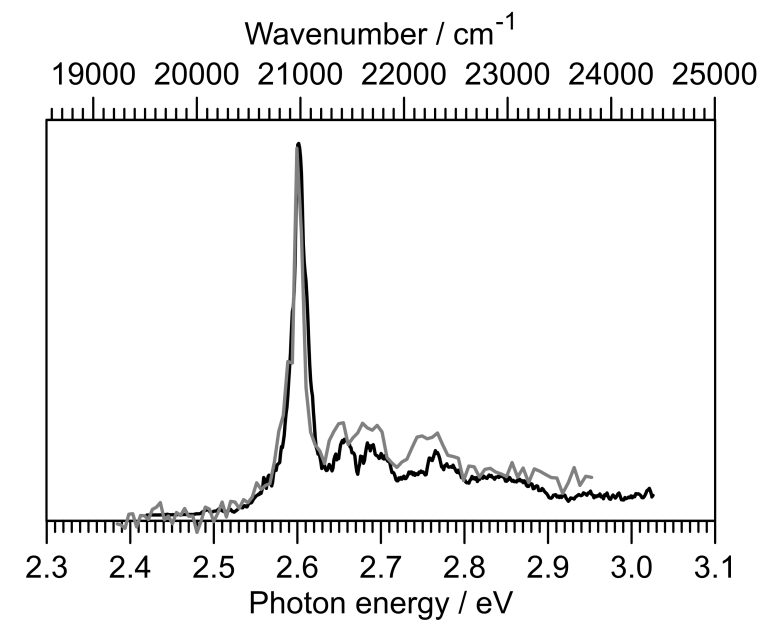

Figure S1: Photodepletion action spectrum for $\mathrm{Py}^{-}$recorded using the Sep1 apparatus (gray). The photdetachment action spectrum from the paper (black) is shown for comparison. 


\section{Time-resolved photoelectron imaging}

Photoelectron spectroscopy was performed using an instrument that combines electrospray ionization, ion trapping at $T \approx 300 \mathrm{~K}$, time-of-flight $\mathrm{m} / z$ separation, and velocity-map imaging detection. ${ }^{8,9}$ Electrosprayed $\mathrm{Py} 1^{-}$from methanol was accumulated in a radio frequency ring-electrode ion trap. The trapped anions were unloaded at $333 \mathrm{~Hz}$ into a set of colinear time-of-flight optics that accelerated the ions along a $1.3 \mathrm{~m}$ flight region toward a continuousmode penetrating field velocity-mapping assembly. ${ }^{9}$ Laser light was timed to interact with the mass-selected ion packet at the centre of the velocity-map imaging stack. Ejected electrons were velocity-mapped onto a dual (chevron) multichannel plate detector, followed by a P43 phosphor screen, which was monitored with a charge-coupled device camera. Velocitymap images were accumulated with a $500 \mathrm{~ns}$ multi-channel plate gate. The velocity-mapping resolution was $\frac{\Delta E}{E} \approx 5 \%$, and the electron kinetic energy (eKE) scale was calibrated from the spectrum of $\mathrm{I}^{-}$. Velocity-map image reconstructions used antialiasing and polar onionpeeling algorithms, ${ }^{10}$ providing the photoelectron spectra and associated photoelectron angular distributions quantified by the conventional $\beta_{2}$ anisotropy parameter. ${ }^{11}$

Femtosecond laser pulses were derived from a Spectra-Physics Ti:sapphire oscillator and regenerative amplifier. The $h \nu=2.60 \mathrm{eV}(477 \pm 5 \mathrm{~nm}, \approx 20 \mu \mathrm{J})$ pump pulses were produced by fourth-harmonic generation (two successive BBO crystals) of the idler output from an optical parametric amplifier (Light Conversion TOPAS-C). The $h \nu=1.55 \mathrm{eV}(800 \mathrm{~nm}, \approx 100 \mu \mathrm{J})$ probe pulse corresponds to the fundamental output of the femtosecond laser. Pump and probe pulses were delayed relative to each other $(\Delta t)$ using a motorised delay line. The pump and probe pulses were combined colinearly using a dichroic mirror and were loosely focused into the interaction region using a curved metal mirror. The pump-probe cross correlation was $\approx 60 \mathrm{fs}$. The cross-correlation was measured in a non-linear crystal and is consistent with fits to the data.

An additional experiment following the procedure detailed in Ref. 12 was performed to determine if there was any thermionic emission signal in the photoelectron spectra at 
$h \nu=2.60 \mathrm{eV}$. The experiment involved delaying the electronic gate applied to the multichannel plate detector by several nanosecond relative to the femtosecond laser pulse, and found that all photoelectron signal disappeared. This measurement confirmed that there is no $\mathrm{S}_{1} \rightarrow \mathrm{S}_{0}$ internal conversion followed by thermionic emission occurring over the nanosecond to millisecond timescale.

A series of photoelectron spectra recorded over the $h \nu=3.1-3.9 \mathrm{eV}$ range are shown in Figure S2. These data were recorded using a Continuum Horizon optical parametric oscillator (2-3 mJ pulse ${ }^{-1}$, loosely focussed to $\left.\approx 5 \mathrm{~mm}^{2}\right)$ pumped by a Continuum Surelite II Nd:YAG laser. The photon energy range is well beyond the $\mathrm{S}_{1}$ band, allowing for clear observation of the direct photodetachment feature (DD in Figure 2a in the paper). Averaged $\beta_{2}$ values over the direct detachment feature are $\beta_{2} \approx-0.8$ across the photon energy range. The loweKE feature in these spectra is presumably due to an indirect electron detachment process associated with exciting $\mathrm{S}_{n}(n>1)$ electronic states. 


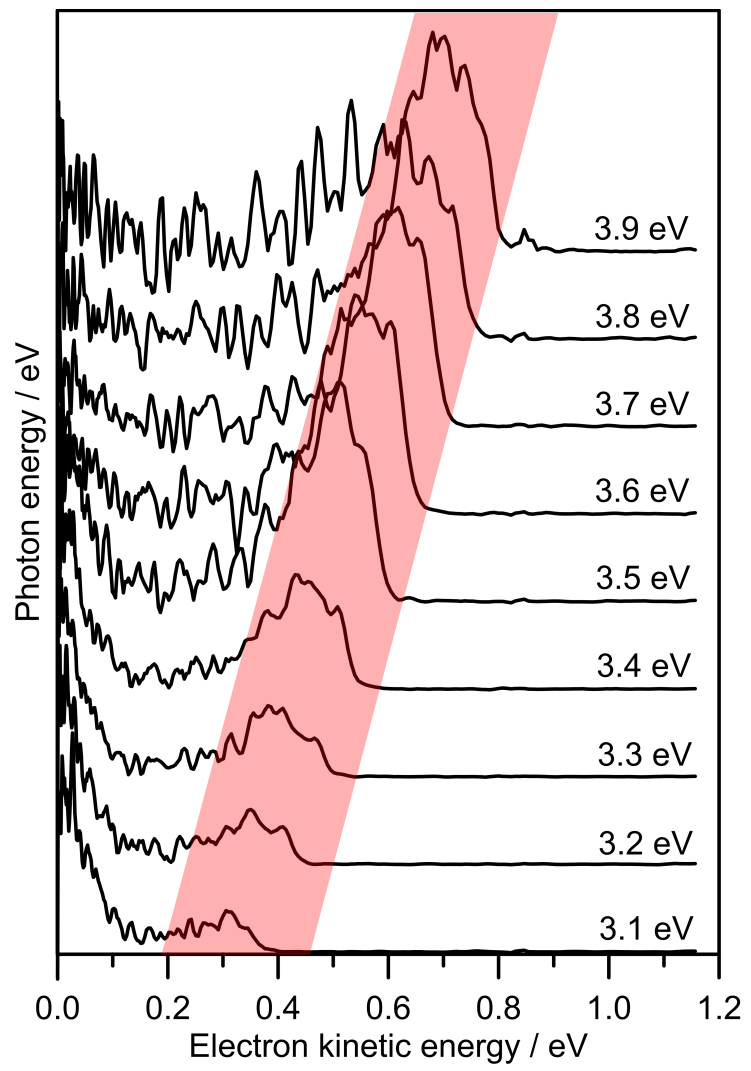

Figure S2: Frequency-resolved photoelectron spectra for Py1 ${ }^{-}$. The red shaded area indicates the direct photodetachment (DD) feature. 


\section{Theoretical}

\section{Geometries and excited states}

Electronic structure calculations were performed using the Gaussian 16.B01 ${ }^{13}$ and CFOUR $2.1^{14}$ software packages. Equilibrium structures and vibrational frequencies were first optimized at the $\omega$ B97X-D/aug-cc-pVDZ level of theory. ${ }^{15,16}$ Franck-Condon-Herzberg-Teller (FCHT) simulation of the $\mathrm{S}_{1} \leftarrow \mathrm{S}_{0}$ absorption profile were performed using the FCHT module as implemented in Gaussian 16.B01. ${ }^{17}$

The DBS was characterized at the EA-EOM-CCSD/aug-cc-pVDZ $+6 s p d$ level of theory (MP2/aug-cc-pVDZ optimized geometry of $\mathrm{Py}^{-}$), where 6 spd indicates addition of eight uncontracted $s, p$ and $d$ functions centered $3 \AA$ beyond the carbon atoms and in the direction of the positive end of the electric dipole moment associated with the radical neutral (Py1). Following the recommendations from Simons and co-workers, ${ }^{18}$ orbital exponents for the additional functions start at $\zeta=0.1$ and follow an even-tempered geometric progression with factor 3.2. Convergence of the computed DBS binding energy with $N$ additional sets of spd functions is shown in Figure S3. Linear dependence and wavefunction convergence problems arose for $N>8$. The calculated ADE is $2.42 \mathrm{eV}$ at the CCSD(T)/aug-cc-pVDZ level of theory, ${ }^{19}$ which is close to the experimental value of $2.4772 \pm 0.0004 \mathrm{eV}$ from Ref. 20 . 


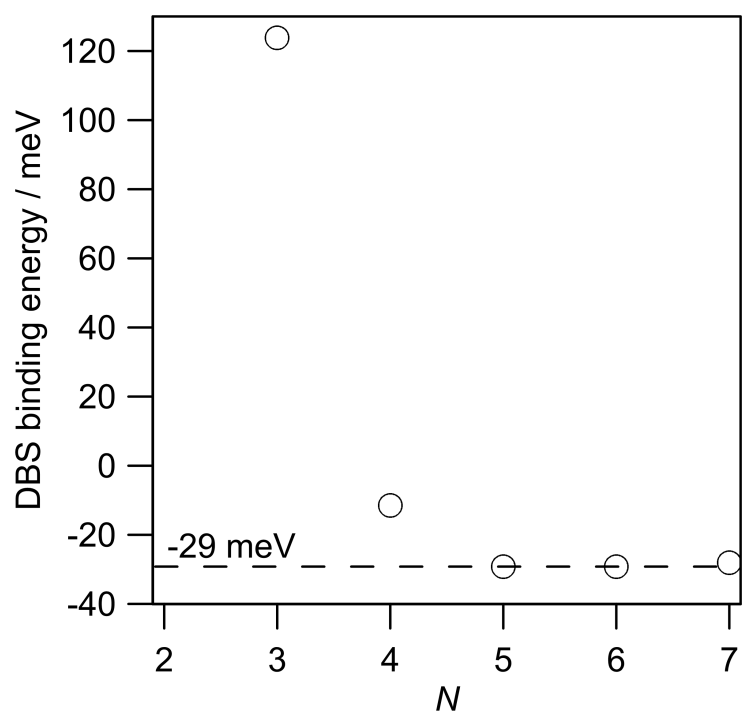

Figure S3: Convergence of the DBS binding energy to $-29 \mathrm{meV}$ (negative indicates a bound state with respect to the detachment continuum) with $N$ additional sets of spd diffuse functions to the aug-cc-pVDZ basis set. The experimental binding energy is $32 \pm 6 \mathrm{meV}$. 


\section{Calculated vibrational frequencies}

Calculated vibrational frequencies for the $S_{0}$ and $S_{1}$ electronic states of Py1- and the $D_{0}$ electronic state of Py1 at the $\omega$ B97X-D/aug-cc-pVDZ level of theory are given in Table S1, $\mathrm{S} 2$ and S3, respectively. The modes are ordered according to the Mulliken scheme for $\mathrm{C}_{s}$ point group symmetry.

The DBS vibrations $\mathbf{4}$ and $\mathbf{7}$ identified in Figure 2a in the paper are associated with modes $47\left(422 \mathrm{~cm}^{-1}, \mathrm{~A}^{\prime}\right)$ and $44\left(524 \mathrm{~cm}^{-1}, \mathrm{~A}^{\prime}\right)$ from Table S3. 
Table S1: Calculated vibrational frequencies for the $\mathrm{S}_{0}$ electronic state of $\mathrm{Py}^{-}$.

\begin{tabular}{|c|c|c|c|c|c|}
\hline Mode & Frequency $/ \mathrm{cm}^{-1}$ & Symmetry & Mode & Frequency $/ \mathrm{cm}^{-1}$ & Symmetry \\
\hline 1 & 3208 & $\mathrm{~A}^{\prime}$ & 37 & 920 & $\mathrm{~A}^{\prime}$ \\
\hline 2 & 3195 & $\mathrm{~A}^{\prime}$ & 38 & 833 & $\mathrm{~A}^{\prime}$ \\
\hline 3 & 3191 & $\mathrm{~A}^{\prime}$ & 39 & 780 & $\mathrm{~A}^{\prime}$ \\
\hline 4 & 3186 & $\mathrm{~A}^{\prime}$ & 40 & 723 & $\mathrm{~A}^{\prime}$ \\
\hline 5 & 3180 & $\mathrm{~A}^{\prime}$ & 41 & 693 & $\mathrm{~A}^{\prime}$ \\
\hline 6 & 3173 & $\mathrm{~A}^{\prime}$ & 42 & 618 & $\mathrm{~A}^{\prime}$ \\
\hline 7 & 3163 & $\mathrm{~A}^{\prime}$ & 43 & 568 & $\mathrm{~A}^{\prime}$ \\
\hline 8 & 3157 & $\mathrm{~A}^{\prime}$ & 44 & 528 & $\mathrm{~A}^{\prime}$ \\
\hline 9 & 3146 & $\mathrm{~A}^{\prime}$ & 45 & 491 & $\mathrm{~A}^{\prime}$ \\
\hline 10 & 1682 & $\mathrm{~A}^{\prime}$ & 46 & 465 & $\mathrm{~A}^{\prime}$ \\
\hline 11 & 1662 & $\mathrm{~A}^{\prime}$ & 47 & 424 & $\mathrm{~A}^{\prime}$ \\
\hline 12 & 1656 & $\mathrm{~A}^{\prime}$ & 48 & 392 & $\mathrm{~A}^{\prime}$ \\
\hline 13 & 1638 & $\mathrm{~A}^{\prime}$ & 49 & 280 & $\mathrm{~A}^{\prime}$ \\
\hline 14 & 1610 & $\mathrm{~A}^{\prime}$ & 50 & 1004 & $A^{\prime \prime}$ \\
\hline 15 & 1574 & $\mathrm{~A}^{\prime}$ & 51 & 979 & $\mathrm{~A}^{\prime \prime}$ \\
\hline 16 & 1536 & $\mathrm{~A}^{\prime}$ & 52 & 969 & $\mathrm{~A}^{\prime \prime}$ \\
\hline 17 & 1507 & $\mathrm{~A}^{\prime}$ & 53 & 956 & $A^{\prime \prime}$ \\
\hline 18 & 1499 & $\mathrm{~A}^{\prime}$ & 54 & 878 & $\mathrm{~A}^{\prime \prime}$ \\
\hline 19 & 1450 & $\mathrm{~A}^{\prime}$ & 55 & 871 & $A^{\prime \prime}$ \\
\hline 20 & 1441 & $\mathrm{~A}^{\prime}$ & 56 & 860 & $\mathrm{~A}^{\prime \prime}$ \\
\hline 21 & 1425 & $\mathrm{~A}^{\prime}$ & 57 & 837 & $A^{\prime \prime}$ \\
\hline 22 & 1411 & $\mathrm{~A}^{\prime}$ & 58 & 787 & $\mathrm{~A}^{\prime \prime}$ \\
\hline 23 & 1384 & $\mathrm{~A}^{\prime}$ & 59 & 755 & $\mathrm{~A}^{\prime \prime}$ \\
\hline 24 & 1338 & $\mathrm{~A}^{\prime}$ & 60 & 736 & $A^{\prime \prime}$ \\
\hline 25 & 1296 & $\mathrm{~A}^{\prime}$ & 61 & 694 & $\mathrm{~A}^{\prime \prime}$ \\
\hline 26 & 1258 & $\mathrm{~A}^{\prime}$ & 62 & 658 & $\mathrm{~A}^{\prime \prime}$ \\
\hline 27 & 1249 & $\mathrm{~A}^{\prime}$ & 63 & 558 & $\mathrm{~A}^{\prime \prime}$ \\
\hline 28 & 1234 & $\mathrm{~A}^{\prime}$ & 64 & 523 & $\mathrm{~A}^{\prime \prime}$ \\
\hline 29 & 1193 & $\mathrm{~A}^{\prime}$ & 65 & 520 & $\mathrm{~A}^{\prime \prime}$ \\
\hline 30 & 1179 & $\mathrm{~A}^{\prime}$ & 66 & 450 & $\mathrm{~A}^{\prime \prime}$ \\
\hline 31 & 1153 & $\mathrm{~A}^{\prime}$ & 67 & 306 & $A^{\prime \prime}$ \\
\hline 32 & 1151 & $\mathrm{~A}^{\prime}$ & 68 & 269 & $\mathrm{~A}^{\prime \prime}$ \\
\hline 33 & 1120 & $\mathrm{~A}^{\prime}$ & 69 & 213 & $\mathrm{~A}^{\prime \prime}$ \\
\hline 34 & 1115 & $\mathrm{~A}^{\prime}$ & 70 & 197 & $\mathrm{~A}^{\prime \prime}$ \\
\hline 35 & 1087 & $\mathrm{~A}^{\prime}$ & 71 & 111 & $\mathrm{~A}^{\prime \prime}$ \\
\hline 36 & 993 & $\mathrm{~A}^{\prime}$ & 72 & 90 & $A^{\prime \prime}$ \\
\hline
\end{tabular}


Table S2: Calculated vibrational frequencies for the $\mathrm{S}_{1}$ electronic state of $\mathrm{Py}^{-}$.

\begin{tabular}{|c|c|c|c|c|c|}
\hline Mode & Frequency $/ \mathrm{cm}^{-1}$ & Symmetry & Mode & Frequency $/ \mathrm{cm}^{-1}$ & Symmetry \\
\hline 1 & 3223 & $\mathrm{~A}^{\prime}$ & 37 & 900 & $\mathrm{~A}^{\prime}$ \\
\hline 2 & 3200 & $\mathrm{~A}^{\prime}$ & 38 & 820 & $\mathrm{~A}^{\prime}$ \\
\hline 3 & 3195 & $\mathrm{~A}^{\prime}$ & 39 & 741 & $\mathrm{~A}^{\prime}$ \\
\hline 4 & 3187 & $\mathrm{~A}^{\prime}$ & 40 & 711 & $\mathrm{~A}^{\prime}$ \\
\hline 5 & 3180 & $\mathrm{~A}^{\prime}$ & 41 & 655 & $\mathrm{~A}^{\prime}$ \\
\hline 6 & 3170 & $\mathrm{~A}^{\prime}$ & 42 & 619 & $\mathrm{~A}^{\prime}$ \\
\hline 7 & 3163 & $\mathrm{~A}^{\prime}$ & 43 & 566 & $\mathrm{~A}^{\prime}$ \\
\hline 8 & 3162 & $\mathrm{~A}^{\prime}$ & 44 & 499 & $\mathrm{~A}^{\prime}$ \\
\hline 9 & 3161 & $\mathrm{~A}^{\prime}$ & 45 & 485 & $\mathrm{~A}^{\prime}$ \\
\hline 10 & 1647 & $\mathrm{~A}^{\prime}$ & 46 & 462 & $\mathrm{~A}^{\prime}$ \\
\hline 11 & 1602 & $\mathrm{~A}^{\prime}$ & 47 & 417 & $\mathrm{~A}^{\prime}$ \\
\hline 12 & 1590 & $\mathrm{~A}^{\prime}$ & 48 & 363 & $\mathrm{~A}^{\prime}$ \\
\hline 13 & 1581 & $\mathrm{~A}^{\prime}$ & 49 & 282 & $\mathrm{~A}^{\prime}$ \\
\hline 14 & 1530 & $\mathrm{~A}^{\prime}$ & 50 & 943 & $A^{\prime \prime}$ \\
\hline 15 & 1509 & $\mathrm{~A}^{\prime}$ & 51 & 937 & $\mathrm{~A}^{\prime \prime}$ \\
\hline 16 & 1495 & $\mathrm{~A}^{\prime}$ & 52 & 923 & $\mathrm{~A}^{\prime \prime}$ \\
\hline 17 & 1467 & $\mathrm{~A}^{\prime}$ & 53 & 884 & $A^{\prime \prime}$ \\
\hline 18 & 1454 & $\mathrm{~A}^{\prime}$ & 54 & 851 & $\mathrm{~A}^{\prime \prime}$ \\
\hline 19 & 1430 & $\mathrm{~A}^{\prime}$ & 55 & 842 & $A^{\prime \prime}$ \\
\hline 20 & 1418 & $\mathrm{~A}^{\prime}$ & 56 & 802 & $\mathrm{~A}^{\prime \prime}$ \\
\hline 21 & 1403 & $\mathrm{~A}^{\prime}$ & 57 & 785 & $A^{\prime \prime}$ \\
\hline 22 & 1362 & $\mathrm{~A}^{\prime}$ & 58 & 756 & $\mathrm{~A}^{\prime \prime}$ \\
\hline 23 & 1326 & $\mathrm{~A}^{\prime}$ & 59 & 717 & $\mathrm{~A}^{\prime \prime}$ \\
\hline 24 & 1314 & $\mathrm{~A}^{\prime}$ & 60 & 693 & $A^{\prime \prime}$ \\
\hline 25 & 1251 & $\mathrm{~A}^{\prime}$ & 61 & 659 & $\mathrm{~A}^{\prime \prime}$ \\
\hline 26 & 1249 & $\mathrm{~A}^{\prime}$ & 62 & 625 & $\mathrm{~A}^{\prime \prime}$ \\
\hline 27 & 1235 & $\mathrm{~A}^{\prime}$ & 63 & 525 & $\mathrm{~A}^{\prime \prime}$ \\
\hline 28 & 1214 & $\mathrm{~A}^{\prime}$ & 64 & 512 & $\mathrm{~A}^{\prime \prime}$ \\
\hline 29 & 1195 & $\mathrm{~A}^{\prime}$ & 65 & 487 & $\mathrm{~A}^{\prime \prime}$ \\
\hline 30 & 1177 & $\mathrm{~A}^{\prime}$ & 66 & 368 & $\mathrm{~A}^{\prime \prime}$ \\
\hline 31 & 1148 & $\mathrm{~A}^{\prime}$ & 67 & 302 & $A^{\prime \prime}$ \\
\hline 32 & 1122 & $\mathrm{~A}^{\prime}$ & 68 & 249 & $\mathrm{~A}^{\prime \prime}$ \\
\hline 33 & 1114 & $\mathrm{~A}^{\prime}$ & 69 & 205 & $\mathrm{~A}^{\prime \prime}$ \\
\hline 34 & 1083 & $\mathrm{~A}^{\prime}$ & 70 & 181 & $\mathrm{~A}^{\prime \prime}$ \\
\hline 35 & 994 & $\mathrm{~A}^{\prime}$ & 71 & 99 & $\mathrm{~A}^{\prime \prime}$ \\
\hline 36 & 979 & $\mathrm{~A}^{\prime}$ & 72 & 84 & $A^{\prime \prime}$ \\
\hline
\end{tabular}


Table S3: Calculated vibrational frequencies for the $\mathrm{D}_{0}$ electronic state of Py1 (radical neutral).

\begin{tabular}{cccccc}
\hline Mode & Frequency $/ \mathrm{cm}^{-1}$ & Symmetry & Mode & Frequency $/ \mathrm{cm}^{-1}$ & Symmetry \\
\hline 1 & 3229 & $\mathrm{~A}^{\prime}$ & 37 & 917 & $\mathrm{~A}^{\prime}$ \\
2 & 3226 & $\mathrm{~A}^{\prime}$ & 38 & 834 & $\mathrm{~A}^{\prime}$ \\
3 & 3223 & $\mathrm{~A}^{\prime}$ & 39 & 780 & $\mathrm{~A}^{\prime}$ \\
4 & 3212 & $\mathrm{~A}^{\prime}$ & 40 & 716 & $\mathrm{~A}^{\prime}$ \\
5 & 3205 & $\mathrm{~A}^{\prime}$ & 41 & 686 & $\mathrm{~A}^{\prime}$ \\
6 & 3202 & $\mathrm{~A}^{\prime}$ & 42 & 617 & $\mathrm{~A}^{\prime}$ \\
7 & 3197 & $\mathrm{~A}^{\prime}$ & 43 & 568 & $\mathrm{~A}^{\prime}$ \\
8 & 3195 & $\mathrm{~A}^{\prime}$ & 44 & 524 & $\mathrm{~A}^{\prime}$ \\
9 & 3191 & $\mathrm{~A}^{\prime}$ & 45 & 489 & $\mathrm{~A}^{\prime}$ \\
10 & 1673 & $\mathrm{~A}^{\prime}$ & 46 & 461 & $\mathrm{~A}^{\prime}$ \\
11 & 1655 & $\mathrm{~A}^{\prime}$ & 47 & 422 & $\mathrm{~A}^{\prime}$ \\
12 & 1636 & $\mathrm{~A}^{\prime}$ & 48 & 386 & $\mathrm{~A}^{\prime}$ \\
13 & 1631 & $\mathrm{~A}^{\prime}$ & 49 & 282 & $\mathrm{~A}^{\prime}$ \\
14 & 1607 & $\mathrm{~A}^{\prime}$ & 50 & 1010 & $\mathrm{~A}^{\prime \prime}$ \\
15 & 1557 & $\mathrm{~A}^{\prime}$ & 51 & 1008 & $\mathrm{~A}^{\prime \prime}$ \\
16 & 1534 & $\mathrm{~A}^{\prime}$ & 52 & 998 & $\mathrm{~A}^{\prime \prime}$ \\
17 & 1516 & $\mathrm{~A}^{\prime}$ & 53 & 979 & $\mathrm{~A}^{\prime \prime}$ \\
18 & 1489 & $\mathrm{~A}^{\prime}$ & 54 & 927 & $\mathrm{~A}^{\prime \prime}$ \\
19 & 1452 & $\mathrm{~A}^{\prime}$ & 55 & 882 & $\mathrm{~A}^{\prime \prime}$ \\
20 & 1448 & $\mathrm{~A}^{\prime}$ & 56 & 874 & $\mathrm{~A}^{\prime \prime}$ \\
21 & 1432 & $\mathrm{~A}^{\prime}$ & 57 & 856 & $\mathrm{~A}^{\prime \prime}$ \\
22 & 1400 & $\mathrm{~A}^{\prime}$ & 58 & 822 & $\mathrm{~A}^{\prime \prime}$ \\
23 & 1369 & $\mathrm{~A}^{\prime}$ & 59 & 771 & $\mathrm{~A}^{\prime \prime}$ \\
24 & 1348 & $\mathrm{~A}^{\prime}$ & 60 & 751 & $\mathrm{~A}^{\prime \prime}$ \\
25 & 1315 & $\mathrm{~A}^{\prime}$ & 61 & 686 & $\mathrm{~A}^{\prime \prime}$ \\
26 & 1257 & $\mathrm{~A}^{\prime}$ & 62 & 647 & $\mathrm{~A}^{\prime \prime}$ \\
27 & 1240 & $\mathrm{~A}^{\prime}$ & 63 & 550 & $\mathrm{~A}^{\prime \prime}$ \\
28 & 1227 & $\mathrm{~A}^{\prime}$ & 64 & 518 & $\mathrm{~A}^{\prime \prime}$ \\
29 & 1207 & $\mathrm{~A}^{\prime}$ & 65 & 500 & $\mathrm{~A}^{\prime \prime}$ \\
30 & 1187 & $\mathrm{~A}^{\prime}$ & 66 & 428 & $\mathrm{~A}^{\prime \prime}$ \\
31 & 1159 & $\mathrm{~A}^{\prime}$ & 67 & 308 & $\mathrm{~A}^{\prime \prime}$ \\
32 & 1151 & $\mathrm{~A}^{\prime}$ & 68 & 262 & $\mathrm{~A}^{\prime \prime}$ \\
33 & 1128 & $\mathrm{~A}^{\prime}$ & 69 & 208 & $\mathrm{~A}^{\prime \prime}$ \\
34 & 1123 & $\mathrm{~A}^{\prime}$ & 70 & 192 & $\mathrm{~A}^{\prime \prime}$ \\
35 & 1087 & $\mathrm{~A}^{\prime}$ & 71 & 108 & $\mathrm{~A}^{\prime \prime}$ \\
36 & 992 & $\mathrm{~A}^{\prime}$ & 72 & 87 & $\mathrm{~A}^{\prime \prime}$ \\
\hline & & & & & \\
\hline
\end{tabular}




\section{FCHT active vibrations}

The predominant $S_{1}$ state vibrations responsible for the simulated vibronic structure in the absorption spectrum in Figure 2b in the paper are shown in Figure S4.

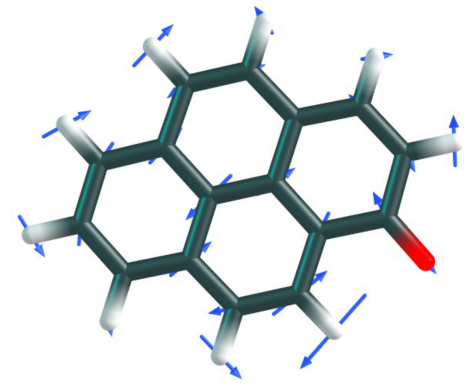

10: $1647 \mathrm{~cm}^{-1}$

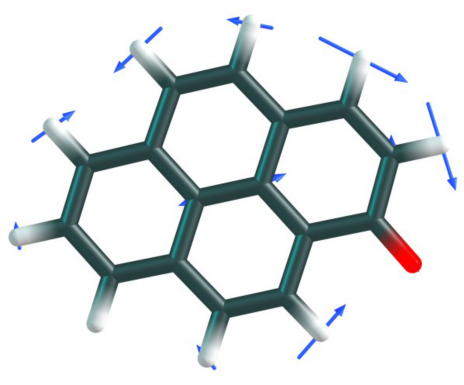

21: $1403 \mathrm{~cm}^{-1}$

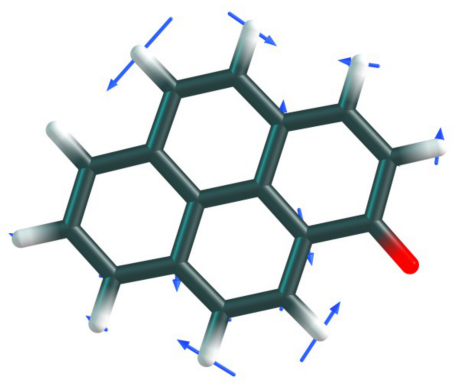

23: $1326 \mathrm{~cm}^{-1}$

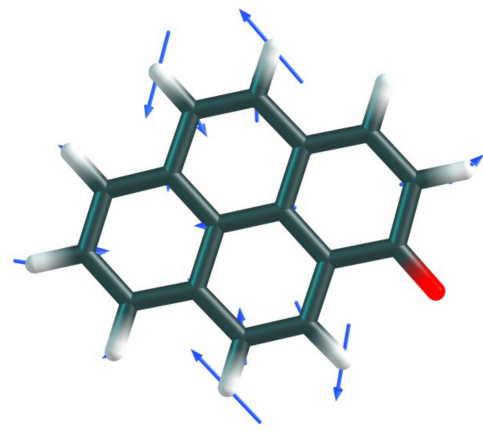

38: $820 \mathrm{~cm}^{-1}$

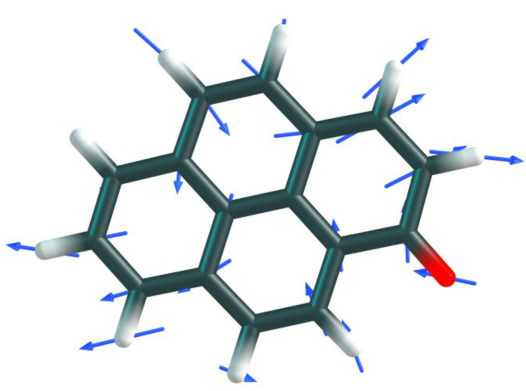

47: $417 \mathrm{~cm}^{-1}$

Figure S4: Principal Franck-Condon active vibrational modes in the simulated $\mathrm{S}_{1} \leftarrow \mathrm{S}_{0}$ absorption spectrum for $\mathrm{Py}^{-}$. Each mode involves in-plane atomic displacements $\left(\mathrm{A}^{\prime}\right.$ symmetry). 
FCHT simulations of the $\mathrm{S}_{1} \leftarrow \mathrm{S}_{0}$ absorption spectrum for Py1- assuming average vibrational temperatures of $T=0 \mathrm{~K}$ and $T=300 \mathrm{~K}$ are shown in Figure S5. The difference between the two simulations is the hot band signal at $T=300 \mathrm{~K}$.
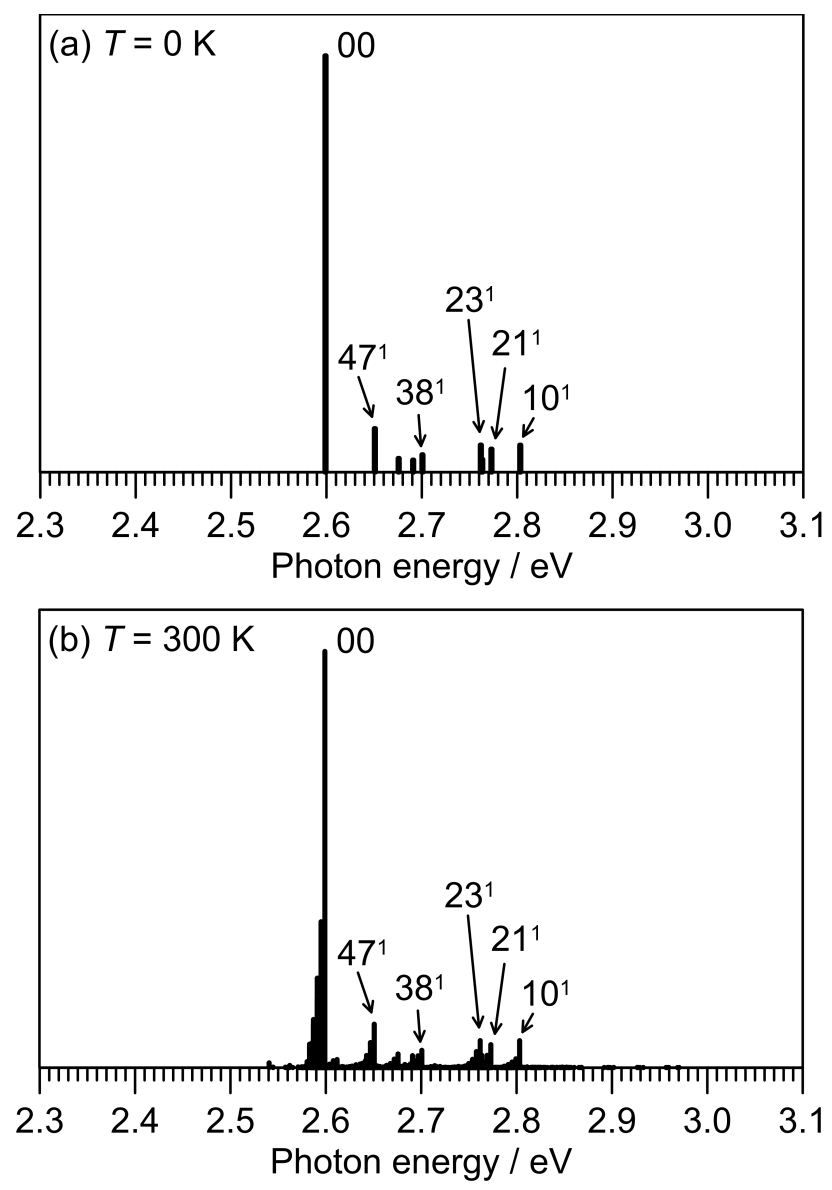

Figure S5: FCHT simulations of the $\mathrm{S}_{1} \leftarrow \mathrm{S}_{0}$ absorption spectrum for $\mathrm{Py}^{-}$at: (a) $T=0 \mathrm{~K}$, (b) $T=300 \mathrm{~K}$. 


\section{References}

(1) Adamson, B. D.; Coughlan, N. J. A.; Markworth, P. B.; Continetti, R. E.; Bieske, E. J. An Ion Mobility Mass Spectrometer for Investigating Photoisomerization and Photodissociation of Molecular Ions. Rev. Sci. Instr. 2014, 85, 123109.

(2) Bull, J. N.; Buntine, J. T.; Scholz, M. S.; Carrascosa, E.; Giacomozzi, L.; Stockett, M. H.; Bieske, E. J. Photodetachment and Photoreactions of Substituted Naphthalene Anions in a Tandem Ion Mobility Spectrometer. Faraday Discuss. 2019, 217, $34-46$.

(3) Bull, J. N.; West, C. W.; Verlet, J. R. R. On the Formation of Anions: Frequency-, Angle-, and Time-Resolved Photoelectron Imaging of the Menadione Radical Anion. Chem. Sci. 2015, 6, 1578-1589.

(4) Bull, J. N.; Scholz, M. S.; Carrascosa, E.; da Silva, G.; Bieske, E. J. Double Molecular Photoswitch Driven by Light and Collisions. Phys. Rev. Lett. 2018, 120, 223002.

(5) Bull, J. N.; da Silva, G.; Scholz, M. S.; Carrascosa, E.; Bieske, E. J. Photoinitiated Intramolecular Proton Transfer in Deprotonated para-Coumaric Acid. J. Phys. Chem. A 2019, 123, 4419-4430.

(6) Støchkel, K.; Milne, B. F.; Nielsen, S. B. Absorption Spectrum of the Firefly Luciferin Anion Isolated in Vacuo. J. Phys. Chem. A 2011, 115, 2155-2159.

(7) Wyer, J. A.; Brøndsted Nielsen, S. Absorption by Isolated Ferric Heme Nitrosyl Cations In Vacuo. Angew. Chem. Int. Ed. 2012, 51, 10256-10260.

(8) Roberts, G. M.; Lecointre, J.; Horke, D. A.; Verlet, J. R. R. Spectroscopy and Dynamics of the 7,7,8,8-tetracyanoquinodimethane Radical Anion. Phys. Chem. Chem. Phys. 2010, 12, 6226-6232. 
(9) Horke, D. A.; Roberts, G. M.; Lecointre, J.; Verlet, J. R. R. Velocity-Map Imaging at Low Extraction Fields. Rev. Sci. Instrum. 2012, 83, 063101.

(10) Roberts, G. M.; Nixon, J. L.; Lecointre, J.; Wrede, E.; Verlet, J. R. R. Toward RealTime Charged-Particle Image Reconstruction Using Polar Onion-Peeling. Rev. Sci. Instrum. 2009, 80, 053104.

(11) Zare, R. N. Photoejection Dynamics. Mol. Photochem. 1972, 4, 1-37.

(12) Bull, J. N.; West, C. W.; Verlet, J. R. R. Internal Conversion Outcompetes Autodetachment From Resonances in the Deprotonated Tetracene Anion Continuum. Phys. Chem. Chem. Phys. 2015, 17, 32464-32471.

(13) Frisch, M. J.; Trucks, G. W.; Schlegel, H. B.; Scuseria, G. E.; Robb, M. A.; Cheeseman, J. R.; Scalmani, G.; Barone, V.; Mennucci, B.; Petersson, G. A.; Nakatsuji, H.; Caricato, M.; Li, X.; Hratchian, H. P.; Izmaylov, A. F.; Bloino, J.; Zheng, G.; Sonnenberg, J. L.; Hada, M.; Ehara, M.; Toyota, K.; Fukuda, R.; Hasegawa, J.; Ishida, M.; Nakajima, T.; Honda, Y.; Kitao, O.; Nakai, H.; Vreven, T.; Montgomery, J. A., Jr.; Peralta, J. E.; Ogliaro, F.; Bearpark, M.; Heyd, J. J.; Brothers, E.; Kudin, K. N.; Staroverov, V. N.; Kobayashi, R.; Normand, J.; Raghavachari, K.; Rendell, A.; Burant, J. C.; Iyengar, S. S.; Tomasi, J.; Cossi, M.; Rega, N.; Millam, J. M.; Klene, M.; Knox, J. E.; Cross, J. B.; Bakken, V.; Adamo, C.; Jaramillo, J.; Gomperts, R.; Stratmann, R. E.; Yazyev, O.; Austin, A. J.; Cammi, R.; Pomelli, C.; Ochterski, J. W.; Martin, R. L.; Morokuma, K.; Zakrzewski, V. G.; Voth, G. A.; Salvador, P.; Dannenberg, J. J.; Dapprich, S.; Daniels, A. D.; Farkas, Ö.; Foresman, J. B.; Ortiz, J. V.; Cioslowski, J.; Fox, D. J. Gaussian 16 Revision B.01. Gaussian Inc. Wallingford CT 2016.

(14) CFOUR, A Quantum Chemical Program Package Written by J. F. Stanton, J. Gauss, L. Cheng, M. E. Harding, D. A. Matthews, P. G. Szalay With Contributions From 
A. A. Auer, R. J. Bartlett, U. Benedikt, C. Berger, D. E. Bernholdt, Y. J. Bomble, O. Christiansen, F. Engel, R. Faber, M. Heckert, O. Heun, C. Huber, T.-C. Jagau, D. Jonsson, J. Jusélius, K. Klein, W. J. Lauderdale, F. Lipparini, T. Metzroth, L. A. Mück, D. P. O’Neill, D.R. Price, E. Prochnow, C. Puzzarini, K. Ruud, F. Schiffmann, W. Schwalbach, C. Simmons, S. Stopkowicz, A. Tajti, J. Vázquez, F. Wang, J.D. Watts and the Integral Packages MOLECULE (J. Almlöf and P. R. Taylor), PROPS (P. R. Taylor), ABACUS (T. Helgaker, H. J. Aa. Jensen, P. Jørgensen, and J. Olsen), and ECP Routines by A. V. Mitin and C. van Wüllen. For the Current Version, see http://www.cfour.de.

(15) Chai, J.-D.; Head-Gordon, M. Long-Range Corrected Hybrid Density Functionals with Damped Atom-Atom Dispersion Corrections. Phys. Chem. Chem. Phys. 2008, 10, $6615-6620$.

(16) Dunning, Jr., T. H. Gaussian Basis Sets for Use in Correlated Molecular Calculations. I. The Atoms Boron Through Neon and Hydrogen. J. Chem. Phys. 1989, 90, 1007.

(17) Santoro, F.; Lami, A.; Improta, R.; Bloino, J.; Barone, V. Effective Method for the Computation of Optical Spectra of Large Molecules at Finite Temperature Including the Duschinsky and Herzberg-Teller Effect: The Qx Band of Porphyrin as a Case Study. J. Chem. Phys. 2008, 128, 224311.

(18) Skurski, P.; Gutowski, M.; Simons, J. How to Choose a One-Electron Basis Set to Reliably Describe a Dipole-Bound Anion. Int. J. Quantum Chem. 2000, 80, 10241038.

(19) Pople, J. A.; Head-Gordon, M.; Raghavachari, K. Quadratic Configuration Interaction. A General Technique For Determining Electron Correlation Energies. J. Chem. Phys. 1987, 87, 5968-5975.

(20) Qian, C.-H.; Zhang, Y.-R.; Yuan, D.-F.; Wang, L.-S. Photodetachment Spectroscopy 
and Resonant Photoelectron Imaging of Cryogenically Cooled 1-pyrenolate. J. Chem.

Phys. 2021, 154, 094308. 